\title{
Electron Current From an RF Microdielectric Barrier Discharge
}

\author{
Jun-Chieh Wang, Napoleon Leoni, Henryk Birecki, Omer Gila, and Mark J. Kushner, Fellow, IEEE
}

\begin{abstract}
Nonarcing microdielectric barrier discharges (mDBDs) using radio-frequency excitation are attractive in that the arrays of devices can be inexpensively produced to generate surface sources of plasma or radicals. Images of the time evolution of electron extraction from an mDBD sustained in atmospheric-pressure $\mathrm{N}_{2}$ are presented.
\end{abstract}

Index Terms-Dielectric barrier discharge, microplasma.

M ICRODIELECTRIC barrier discharges (mDBDs) consist of microplasma devices $(10-100 \mu \mathrm{m}$ in diameter) wherein one or both of the electrodes are covered with a dielectric [1]. In many applications, such as materials processing, the mDBD may be operated at atmospheric pressure driven by radio-frequency (RF) waveforms. Extraction of electron current out of the mDBD plasma requires a third electrode [2]. After the plasma is initiated, charging of the dielectrics may terminate the discharge by reducing the gap voltage below its self-sustaining value. When the polarity of the applied voltage changes, the dielectric surface charges from the previous RF cycle enhance the gap voltage so that the electron avalanche is more intense. At atmospheric pressure, the plasma decay may be only tens of nanoseconds, whereas the RF period may be up to hundreds of nanoseconds. As a result, the microplasma may need to be reignited each RF cycle. In this paper, we present computed images of electrons extracted from an RF mDBD.

The cylindrically symmetric mDBD device is shown in Fig. 1. An RF electrode driven at $1.4 \mathrm{kV}$ at $10 \mathrm{MHz}$ is buried in a printed circuit board. A grounded electrode is separated from the RF electrode by a dielectric sheet $19 \mu \mathrm{m}$ thick with dielectric constant $\varepsilon=20$. The grounded electrode has a cavity $65 \mu \mathrm{m}$ in diameter with a $35-\mu \mathrm{m}$ opening. An extraction electrode biased with $2 \mathrm{kV}$ dc through a $100-\mathrm{k} \Omega$ resistor is separated from the grounded electrode by a $475-\mu \mathrm{m}$ gap. The gas is $\mathrm{N}_{2}$ at $1 \mathrm{~atm}$. The model we used, nonPDPSIM, is a 2-D simulation performed on an unstructured mesh in which Poisson's, continuity, and surface charge balance equations are solved for electric potential and transport of neutral and charged species [3]. Radiation transport is addressed by a Green's function propagator. A Monte Carlo simulation tracks the ionization

Manuscript received December 1, 2010; revised March 4, 2011; accepted March 6, 2011. Date of publication April 21, 2011; date of current version November 9, 2011. This work was supported by the Hewlett Packard Research Labs.

J.-C. Wang and M. J. Kushner are with the Department of Electrical Engineering and Computer Science, University of Michigan, Ann Arbor, MI 48109-2122 USA (e-mail: junchwan@umich.edu; mjkush@umich.edu).

N. Leoni, H. Birecki, and O. Gila are with the Hewlett Packard Research Labs, Palo Alto, CA 94304-1185 USA (e-mail: napoleon.j.leoni@hp.com; birecki@hpl.hp.com; omer_gila@hp.com).

Digital Object Identifier 10.1109/TPS.2011.2126606

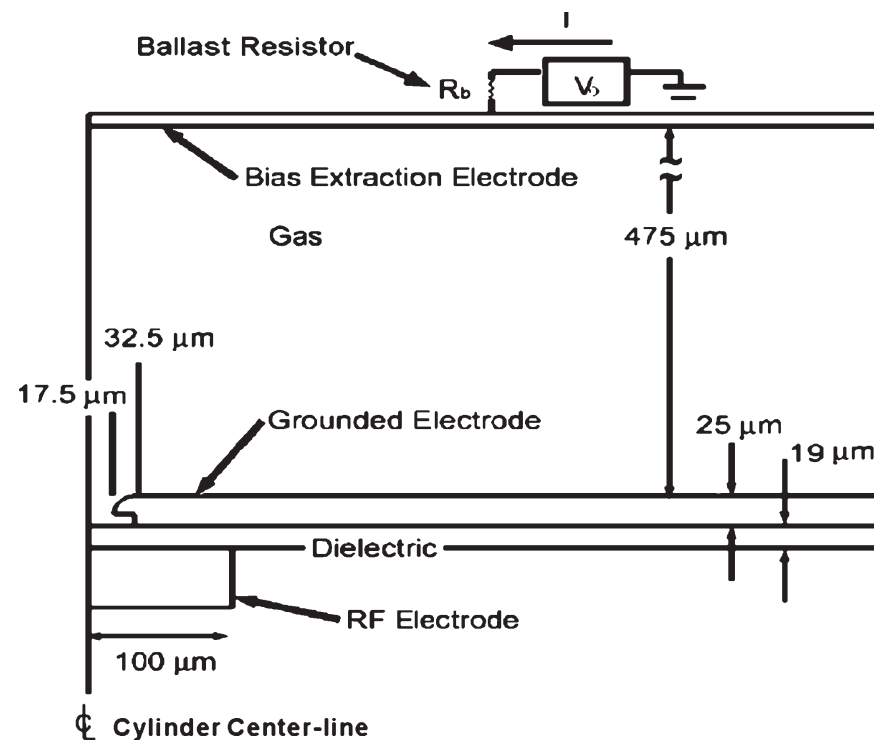

Fig. 1. Schematic of the cylindrical mDBD device.

sources produced by sheath-accelerated secondary electrons. All inelastic processes are accounted for.

The images shown in Fig. 2 are of the time evolution of the electron density and current extraction from the $\mathrm{mDBD}$ cavity during transition to a periodic steady state. The third dimension (height) and contours represent electron density. The lower sequence shows the electron density in the cavity. The upper sequence shows the plume of electrons extracted from the cavity. The electron densities in the cavity are as large as $3 \times 10^{15} \mathrm{~cm}^{-3}$. Ionization is provided roughly equally from bulk electron impact and by the secondary sheath-accelerated electrons. In the plume adjacent to the collection electrode, the densities are up to $7 \times 10^{11} \mathrm{~cm}^{-3}$. There is little ionization in the gap, and so the majority of the current is extracted out of the microdischarge cavity. When the RF voltage crosses from negative to positive ( $275 \mathrm{~ns}$ ), the net voltage across the gap is sufficiently small due to the previous positive charging of the dielectric that the electron plume is directed toward the dielectric and the electron flux charges the dielectric negatively. Before the positive peak RF potential ( $300 \mathrm{~ns}$ ), a small flux of electrons is extracted out of the cavity due to the negative surface charging. As the electron extraction exceeds the ionization rate, the electron plume breaks up in the gap, and the electrons are nearly depleted in the microcavity ( $313 \mathrm{~ns}$ ). When the RF voltage decreases to zero, no external potential is applied; however, there is a large negative potential produced by the surface charges. The plasma is reignited due to the avalanche of the remaining residual electrons in the cavity, and a large electron 


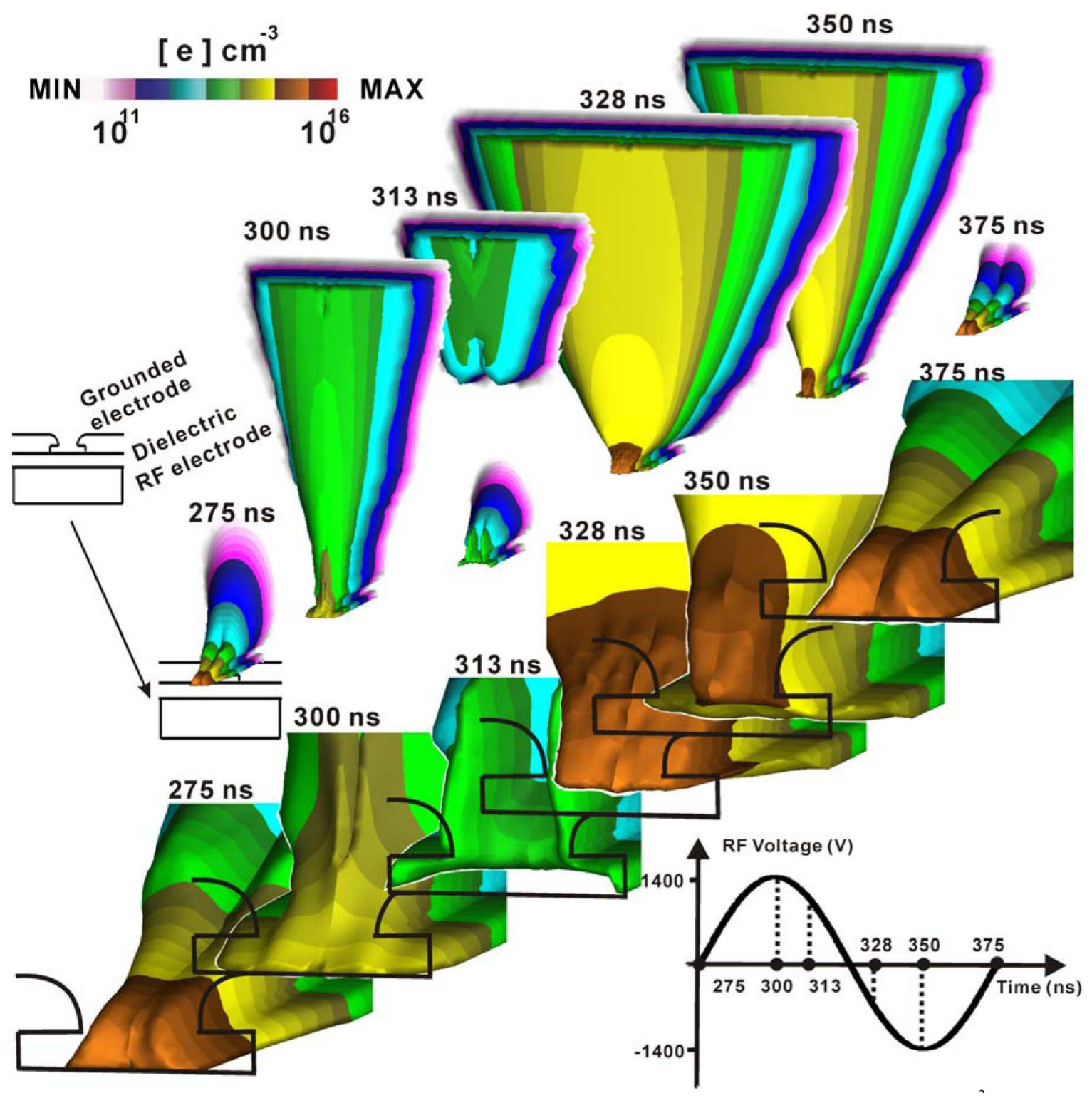

Fig. 2. Time evolution of the electron current extraction from the $\mathrm{mDBD}$ in 1 -atm $\mathrm{N}_{2}$ at $10 \mathrm{MHz}\left(\log \mathrm{scale}, \mathrm{cm}^{-3}\right.$ ). (Top) Electron density in the plume across the gap. (Bottom) Electron density in the microcavity. The third dimension (height) and contours are the electron density on a log scale. The cylindrically symmetric calculation is mirrored across the axis.

current is then extracted to the biased electrode. The electron plume reaches its greatest extent ( $328 \mathrm{~ns}$ ) slightly after the zero crossing of the RF voltage. Before the negative peak of the RF voltage ( $350 \mathrm{~ns}$ ), sufficient positive ions have been collected on the dielectric to reduce the voltage drop across the gap. The electron plume then begins to diminish, and the cycle restarts.

Images for the time evolution of the electron extraction from an RF mDBD have been presented. By controlling the geometry, the gas mixture, and the time-varying voltage, it is feasible to control charge extraction at atmospheric pressure.

\section{REFERENCES}

[1] U. Kogelschatz, "Dielectric-barrier discharges: Their history, discharge physics and industrial applications," Plasma Chem. Plasma Process., vol. 23, no. 1, pp. 1-46, Mar. 2003.

[2] G. Bauville, B. Lacour, L. Magne, V. Puech, J.-P. Boeuf, E. Muños Serrano, and L. C. Pitchford, "Singlet oxygen production in a microcathode sustained discharge," Appl. Phys. Lett., vol. 90, no. 3, p. 031501, Jan. 2007.

[3] N. Y. Babaeva, A. N. Bhoj, and M. J. Kushner, "Streamer dynamics in gases containing dust particles," Plasma Sources Sci. Technol., vol. 15, no. 4, pp. 591-602, Nov. 2006. 